PROCEEDINGS OF THE

AMERICAN MATHEMATICAL SOCIETY

Volume 131, Number 7, Pages 2049-2051

S 0002-9939(03)06819-9

Article electronically published on February 5, 2003

\title{
SMOOTH NORMS ON CERTAIN $C(K)$ SPACES
}

\author{
PETR HÁJEK
}

(Communicated by Jonathan M. Borwein)

\begin{abstract}
C(K)$ spaces admit an equivalent $C^{\infty}$-smooth renorming whenever $K^{\left(\omega_{1}\right)}=\emptyset$.
\end{abstract}

In this note we consider the problem of finding on a given $C(K)$ space an equivalent norm of the highest possible smoothness. It is a classical result (e.g. [DGZ]) that the existence of an equivalent $C^{1}$-smooth norm on a Banach space implies that the space is Asplund. On the other hand, deep examples of Haydon $([\mathrm{H}]$, see also [DGZ]) show that not every $C(K)$ Asplund space admits an equivalent Gâteaux smooth renorming.

So far, an equivalent $C^{\infty}$ renorming was constructed on $C(K)$ spaces where $K^{\left(\omega_{0}\right)}=\emptyset($ GPWZ $]$ ), and a $C^{1}$ norm is guaranteed when $K^{\left(\omega_{1}\right)}=\emptyset([\mathrm{D}])$. Haydon's $C^{\infty}$ renorming techniques work well for certain tree-like compact sets $K$, which may have nonempty derived sets of arbitrary large ordinal number, but their disadvantage is that they put very strong structural restrictions on $K$ (apart from the obvious and necessary scatteredness). This is not accidental, because the abovementioned example of $C(K)$ without a Gâteaux norm has $K^{\left(\omega_{1}\right)}$ a singleton.

In our note we show the existence of $C^{\infty}$ renormings whenever $K^{\left(\omega_{1}\right)}=\emptyset$. This is the best possible result without additional structural assumptions on $K$. However, it is really only a small step towards a desired general theorem linking the existence of $C^{\infty}$ renorming of $C(K)$ to some other properties of the space, such as the existence of a dual LUR renorming of $C(K)$. For background material and notation we refer to DGZ.

Definition 1. Let $S \subset \ell_{\infty}(\Gamma), \Phi: S \rightarrow \mathbb{R}$. We say that $\Phi$ locally depends on finitely many coordinates (LDF) if for every $f \in S$ there exist $\varepsilon>0, \gamma_{1}, \ldots, \gamma_{n} \in \Gamma$ and $F: \mathbb{R}^{n} \rightarrow \mathbb{R}$ such that:

$$
\Phi(g)=F\left(g\left(\gamma_{1}\right), \ldots, g\left(\gamma_{n}\right)\right) \quad \text { whenever } g \in B(f, \varepsilon) \cap S .
$$

Given $1>\delta>0$, find $\phi_{\delta}: \mathbb{R} \rightarrow \mathbb{R}$ such that $\phi_{\delta}$ is $C^{\infty}$-smooth, even and convex, and $\phi_{\delta}([0,1-\delta])=0, \phi_{\delta}(1)=1$.

Definition 2. Let $f \in \ell_{\infty}(\Gamma)$. Put $f^{\infty}=\inf \{t, \operatorname{card}\{\gamma,|f(\gamma)|>t\}$ is finite $\}$.

Received by the editors March 20, 2001.

2000 Mathematics Subject Classification. Primary 46B03, 46B10, 46B20.

The author was supported by grants AV 1019003, A1 019205 and GAČR 201/01/11098. 
Lemma 3. Let $1>\delta>0$, and let $\Phi: \ell_{\infty}(\Gamma) \rightarrow \mathbb{R} \cup\{+\infty\}$ be a convex function defined by

$$
\Phi(f)=\sum_{\gamma \in \Gamma} \phi_{\delta}(f(\gamma))
$$

Then $\Phi$ restricted to $\left\{f \in \ell_{\infty}(\Gamma), f^{\infty}<1-\delta\right\}$ is finite, LDF and $C^{\infty}$-smooth.

Proof. Given $f, f^{\infty}<1-\delta$, the set $\Theta=\left\{\gamma \in \Gamma,|f(\gamma)|>f^{\infty}+\frac{1-\delta-f^{\infty}}{4}\right\}$ is finite. Thus for $g \in B\left(f, \frac{1-\delta-f^{\infty}}{2}\right)$ we have $\Phi(g)=\sum_{\gamma \in \Theta} \phi_{\delta}(g(\gamma))$, which is a finite sum of $C^{\infty}$ smooth convex functions.

Theorem 4. Let $K$ be a scattered compact, $K^{\left(\omega_{1}\right)}=\emptyset$. Then $C(K)$ admits an equivalent $L D F$ and $C^{\infty}$-smooth norm.

Proof. There is $\Lambda<\omega_{1}$ such that $K^{(\Lambda)} \neq \emptyset$ is finite and $K^{(\Lambda+1)}=\emptyset$. The space $C_{0}(K)=\left\{f \in C(K), f\left(K^{(\Lambda)}\right)=0\right\}$ is isomorphic to $C(K)$.

Put $L_{\alpha}=K^{(\alpha)} \backslash K^{(\alpha+1)}, \alpha \leq \Lambda$, and fix $\left\{\delta_{\alpha}\right\}_{\alpha \leq \Lambda}$, such that $\delta_{\alpha}>0$ and $\prod_{\alpha=0}^{\Lambda}\left(1+\delta_{\alpha}\right)<2$. Put $\psi_{\alpha}=\phi_{\frac{\delta_{\alpha+1}}{8}}, D_{\alpha}=\prod_{\beta=0}^{\alpha}\left(1+\delta_{\beta}\right)$. Let us define a convex function $\Psi: C_{0}(K) \rightarrow \mathbb{R} \cup\{+\infty\}$ by $\Psi(f)=\sum_{0 \leq \alpha \leq \Lambda} \sum_{\gamma \in L_{\alpha}} \psi_{\alpha}\left(D_{\alpha} \cdot f(\gamma)\right)$.

Our aim will be to show that $\Psi^{-1}\left(\left[0, \frac{1}{2}\right]\right)$ is the unit ball of an equivalent LDF (canonically, $\left.C_{0}(K) \subset \ell_{\infty}(K)\right)$ and $C^{\infty}$ smooth norm on $C_{0}(K)$.

For every $f \in C_{\infty}(K), 0 \leq \alpha \leq \Lambda$ put

$$
a_{\alpha}^{f}=\left(\left.f\right|_{L_{\alpha}}\right)^{\infty}, \quad b_{\alpha}^{f}=\left\|\left.f\right|_{L_{\alpha}}\right\|_{\infty} .
$$

Note that $\alpha \rightarrow D_{\alpha}$ is an increasing and thus upper semicontinuous (usc) function on $[0, \Lambda]$ (which is a compact space when considered with its natural interval topology). We have:

$$
\begin{aligned}
b_{\alpha+1}^{f} & =a_{\alpha}^{f} \quad \text { and } \quad \alpha \rightarrow b_{\alpha}^{f} \in C[0, \Lambda], \\
b_{\alpha}^{f} & =\lim _{\tau / \alpha, \tau<\alpha} a_{\tau}^{f} .
\end{aligned}
$$

Therefore, for a fixed $f \neq 0, \alpha \rightarrow D_{\alpha} \cdot b_{\alpha}^{f}$ is usc on $[0, \Lambda]$, so it attains its maximum $M_{f}$ at some $\beta \in[0, \Lambda)$. Clearly, $M_{f} \geq D_{\alpha+1} b_{\alpha+1}^{f}=\left(1+\delta_{\alpha+1}\right) D_{\alpha} a_{\alpha}^{f}$, and so $D_{\alpha} a_{\alpha}^{f} \leq \frac{M_{f}}{1+\delta_{\alpha+1}} \leq M_{f}\left(1-\frac{\delta_{\alpha+1}}{2}\right)$, for all $\alpha \in[0, \Lambda]$.

First note that $D_{\beta} b_{\beta}^{f}>\left(D_{\alpha} a_{\alpha}^{f}\right)^{\infty}$. Indeed, otherwise there exist increasing sequences $\alpha_{n} \nearrow \gamma, \alpha_{n} \in[0, \Lambda), D_{\alpha_{n}} a_{\alpha_{n}}^{f} \nearrow D_{\beta} b_{\beta}^{f}$. However, by $(*), a_{\alpha_{n}}^{f} \rightarrow b_{\gamma}^{f}, D_{\gamma}>$ $\left(1+\delta_{\gamma}\right) D_{\alpha_{n}}$ for $n \in \mathbb{N}$, and consequently $D_{\gamma} b_{\gamma}^{f} \geq\left(1+\delta_{\gamma}\right) D_{\beta} b_{\beta}^{f}$, a contradiction. So there exists $\varepsilon_{f}>0$, such that $\operatorname{card}\left\{\alpha, D_{\alpha} a_{\alpha}^{f}>D_{\beta} b_{\beta}^{f}-2 \varepsilon_{f}\right\}$ is finite. Next we claim that $\operatorname{card}\left\{\alpha, D_{\alpha} b_{\alpha}^{f}>D_{\beta} b_{\beta}^{f}-\varepsilon_{f}\right\}$ is also finite.

Again, otherwise there exists $\alpha_{n} \nearrow \gamma, D_{\alpha_{n}} b_{\alpha_{n}}^{f}>D_{\beta} b_{\beta}^{f}-\varepsilon_{f}$. Using $(*)$, and passing to a suitable subsequence of $\left\{\alpha_{n}\right\}$ we find $\beta_{n} \nearrow \gamma, \alpha_{n} \leq \beta_{n}<\alpha_{n+1}$ such that

$$
D_{\beta_{n}} \alpha_{\beta_{n}}^{f} \geq D_{\alpha_{n+1}} b_{\alpha_{n+1}}^{f}-\frac{\varepsilon_{f}}{2} .
$$

This is a contradiction with the definition of $\varepsilon_{f}$, since

$$
\left\{\beta_{n}\right\} \subset\left\{\alpha, D_{\alpha} a_{\alpha}^{f}>D_{\beta} \alpha_{\beta}^{f}-2 \varepsilon_{f}\right\} .
$$


Put $O=\left\{f \in C_{0}(K), M_{f}<1\right\} \subset 2 B_{C_{0}(K)}$. We claim that $\left.\Psi\right|_{O}$ is finite, $C^{\infty}$ smooth and LDF. Moreover, $\Psi^{-1}\left(\left[0, \frac{1}{2}\right]\right) \subset \operatorname{int} O$, which by the implicit function theorem (Di]) finishes the proof.

Choose any $f \in O$. Consider the finite set $A=\left\{\alpha \in[0, \Lambda]\right.$, either $D_{\alpha} b_{\alpha}^{f}>$ $M_{f}-\varepsilon_{f}$ or $\left.\delta_{\alpha+1} \geq \frac{\varepsilon_{f}}{4}\right\}$, and put $\delta_{f}=\min \left\{\frac{\delta_{\alpha+1}}{16}, \alpha \in A\right\}$. Let us check that $\left.\Psi\right|_{B\left(f, \delta_{f}\right)}$ depends on finitely many coordinates (therefore it is necessarily $C^{\infty}$-smooth). If $\gamma \in L_{\alpha}, \alpha \notin A$, then $4 \delta_{\alpha+1}<\varepsilon_{f}$ and $D_{\alpha} b_{\alpha}^{f} \leq M_{f}-\varepsilon_{f} \leq 1-\varepsilon_{f} \leq 1-4 \delta_{\alpha+1}$. For $g \in B\left(f, \delta_{f}\right),|g(\gamma)| \leq|f(\gamma)|+\delta_{f}$, so

$$
\left|D_{\alpha} g(\gamma)\right| \leq D_{\alpha} b_{\alpha}^{f}+2 \delta_{f} \leq 1-\varepsilon_{f}+2 \delta_{f} \leq 1-2 \delta_{\alpha+1}
$$

Consequently,

$$
\psi_{\alpha}\left(D_{\alpha} g(\gamma)\right)=0 \quad \text { and } \quad \Psi(g)=\sum_{\alpha \in A} \sum_{\gamma \in L_{\alpha}} \psi_{\alpha}\left(D_{\alpha} g(\gamma)\right)
$$

If $\alpha \in A, \gamma \in L_{\alpha}$, and $|f(\gamma)|<a_{\alpha}^{f}\left(1+\frac{\delta_{\alpha+1}}{8}\right)$, then

$$
\left|D_{\alpha} f(\gamma)\right| \leq D_{\alpha} a_{\alpha}^{f}\left(1+\frac{\delta_{\alpha+1}}{8}\right) \leq M_{f}\left(1-\frac{\delta_{\alpha+1}}{2}\right)\left(1+\frac{\delta_{\alpha+1}}{8}\right) \leq 1-\frac{\delta_{\alpha+1}}{4} .
$$

If $g \in B\left(f, \delta_{f}\right)$, we then have

$$
\left|D_{\alpha} g(\gamma)\right| \leq 1-\frac{\delta_{\alpha+1}}{4}+2 \delta_{f} \leq 1-\frac{\delta_{\alpha+1}}{8}
$$

Consequently, in this case also $\psi_{\alpha}\left(D_{\alpha} g(\gamma)\right)=0$. The remaining set $S=\left\{\gamma, \gamma \in L_{\alpha}\right.$ for $\alpha \in A$ and $\left.f(\gamma) \geq a_{\alpha}^{f}\left(1+\frac{\delta_{\alpha+1}}{8}\right)\right\}$ is clearly finite, and we have

$$
\Psi(g)=\sum_{\alpha \in A} \sum_{\gamma \in S \cap L_{\alpha}}\left(D_{\alpha} g(\gamma)\right)
$$

whenever $g \in B\left(f, \delta_{f}\right)$. This proves (Lemma 3) that $\left.\Psi\right|_{O}$ is $C^{\infty}$-smooth and LDF. It is obvious that $M_{f} \leq \frac{1}{4}$ implies $\Psi(f)=0$ and $M_{f}=1$ implies $\Psi(f) \geq 1$. Thus $B=\Psi^{-1}\left(\left[0, \frac{1}{2}\right]\right)$ is an equivalent unit ball of $C_{0}(K)$. By the implicit function theorem, its Minkowski functional is $C^{\infty}$-smooth and LFD.

\section{REFERENCES}

[D] R. Deville, Problemes de renormages, J. Funct. Anal. 68 (1986), 117-129. MR 87m:46035

[DGZ] R. Deville, G. Godefroy and V. Zizler, Smoothness and renormings in Banach spaces (1993), Longman. MR 94d:46012

[Di] J. Dieudonné, Foundations of Modern Analysis (1969), Acad. Press. MR 50:1782

[GPWZ] G. Godefroy, J. Pelant, J. H. M. Whitfield and V. Zizler, Banach space properties of Cisielski-Pol's C $(K)$, Proc. AMS 103 (1988), 1087-1094. MR 89i:46042

[H] R. Haydon, Trees in renorming theory, Proc. London Math. Soc. 78 (1999), 549-584. MR 2000d:46011

Mathematical Institute, Czech Academy of Science, Žitná 25, Praha, 11567, Czech REPUBLIC

E-mail address: hajek@math.cas.cz 